\title{
Confiabilidade de testes de caminhada em pacientes claudicantes: estudo piloto
}

\author{
Reliability of walking tests in claudicating patients: a pilot study \\ Inácio Teixeira da Cunha-Filho', Danielle Aparecida Gomes Pereira ${ }^{2}$, \\ André Maurício Borges de Carvalho ${ }^{3}$, Leilane Campedeli ${ }^{4}$, \\ Michelle Soares ${ }^{4}$, Joyce de Sousa Freitas ${ }^{4}$
}

\section{Resumo}

Contexto: Uma vez que a obstrução arterial periférica pode se apresentar de maneira difusa, com clínica diversa e com resultados de intervenção variados, é fundamental que a avaliação dos pacientes com doença arterial obstrutiva periférica seja feita com instrumentos que possam apresentar dados objetivos e reprodutíveis.

Objetivo: Investigar e contrastar a confiabilidade do teste de caminhada de 6 minutos (T6M) com teste de deslocamento bidirecional progressivo (TDBP) em indivíduos claudicantes portadores de doença arterial obstrutiva periférica.

Métodos: Quatorze pacientes em estágio II de Fontaine participaram deste estudo piloto. Onze pacientes realizaram ambos os testes e três realizaram apenas T6M. Após familiarização, os pacientes foram avaliados em duas ocasiões distintas com intervalo máximo de 1 semana entre si. O coeficiente de correlação de intraclasse $\left(\mathrm{ICC}_{2,1}\right)$ foi utilizado para avaliação da reprodutibilidade teste-reteste.

Resultados: A média da distância máxima de caminhada no teste e no reteste no T6M foi de 397,04 $\pm 120,74$ e 408,6 6153,64 metros ( $\mathrm{p}=$ $0,58)$, respectivamente, com ICC $=0,87(\mathrm{p}=0,00005)$; já no TDBP, a média foi de $345 \pm 145,75$ metros e, no reteste, de 345,91 $\pm 127,97$ ( $\mathrm{p}=$ $0,92)$, com ICC $=0,99(\mathrm{p}=0,00005)$. O tempo médio para surgimento da dor inicial, em segundos, com o T6M, foi de 172,25 $\pm 88,23$ (teste) e $148,58 \pm 70,36$ (reteste) $(\mathrm{p}=0,13)$, com ICC $=0,81(\mathrm{p}=0,0004)$. No TDBP, o tempo médio foi de $282 \pm 141,90$ (teste) e $267,14 \pm 150,58$ (reteste) $(\mathrm{p}=0,55)$, com ICC $=0,91(\mathrm{p}=0,0008)$.

Conclusão: Ambos os testes de caminhada são confiáveis e úteis para avaliação clínico-funcional desses pacientes. O TDBP, entretanto, gerou índices de confiabilidade mais elevados, podendo ser melhor opção para avaliação da performance desses indivíduos.

Palvras-chave: Confiabilidade dos testes, claudicação intermitente, caminhada.

\begin{abstract}
Background: Considering that peripheral arterial obstruction is a diffuse condition, manifesting as a variable clinical feature and with varied intervention outcomes, it is important to assess patients with peripheral artery occlusive disease using instruments that may provide objective and reliable data.
\end{abstract}

Objective: To investigate and compare the reliability of both the 6-minute walking test (6MWT) and the shuttle walking test (SWT) in patients with claudication secondary to peripheral artery occlusive disease.

Methods: Fourteen Fontaine stage II patients participated in the study. Eleven patients were submitted to both tests, while three patients only performed the 6MWT. After familiarization, the patients were tested on two occasions, with maximal 1-week interval between measurements. The intraclass correlation coefficient $\left(\mathrm{ICC}_{2,1}\right)$ was used to check for test-retest reliability.

Results: Mean maximal walking distance for the 6MWT in both test and retest was $397.04 \pm 120.74$ and $408.6 \pm 153.64 \mathrm{~m}(\mathrm{p}=0.58)$ respectively; ICC $=0.87(\mathrm{p}=0.00005)$. For the SWT, mean was $345 \pm 145.75 \mathrm{~m}$, and $345.91 \pm 127.97 \mathrm{~m}$ in the retest $(\mathrm{p}=0.92) ; \mathrm{ICC}=$ $0.99(\mathrm{p}=0.00005)$. Mean times for claudication onset with the 6MWT test-retest were $172.25 \pm 88.23$ and $148.58 \pm 70.36 \mathrm{~s}(\mathrm{p}=0.13) ; \mathrm{ICC}=$ $0.81(\mathrm{p}=0.0004)$; while for the SWT these values were $282 \pm 141.90$ and $267.14 \pm 150.58 \mathrm{~s}(\mathrm{p}=0.55) ; \mathrm{ICC}=0.91(\mathrm{p}=0.0008)$.

Conclusion: Both walking tests are reliable and can be used for clinical and functional assessment of these patients. The SWT, however, yielded higher reliability coefficients, which suggests that it may be preferable to evaluate these individuals' performance.

Keywords: Reliability test, intermittent claudication, walking.

1. Fisioterapeuta. PhD, Centro Universitário de Belo Horizonte (UNI-BH), Belo Horizonte, MG.

2. Fisioterapeuta. Especialista.

3. Médico angiologista.

4. Fisioterapeuta.

Apresentado em forma de banner e publicado nos anais do XV Congresso Brasileiro de Geriatria e Gerontologia: II Jornada Goiânia de Geriatria e Gerontologia e IV Encontro Nacional de Ligas Acadêmicas de Geriatria e Gerontologia, realizado no Centro de Convenção de Goiânia, de 6 a 9 de junho de 2006.

Não foram declarados conflitos de interesse associados à publicação deste artigo.

Artigo submetido em 29.08.07, aceito em 15.04.08.

J Vasc Bras. 2008;7(2):106-111.

Copyright $\odot 2008$ by Sociedade Brasileira de Angiologia e de Cirurgia Vascular 


\section{Introdução}

A doença arterial oclusiva periférica (DAOP) é uma patologia caracterizada por perfusão inadequada secundária à obstrução arterial que acomete principalmente membros inferiores. No Brasil, 5,3\% dos indivíduos acima de 45 anos apresentam alta probabilidade de desenvolver DAOP ${ }^{1}$. A prevalência da doença é inferior a $2 \%$ para homens com menos de 50 anos, aumentando para mais de $5 \%$ naqueles com idade superior a 70 anos $^{2}$.

A claudicação intermitente (CI) é um sintoma usual na DAOP, que se manifesta comumente como dor, cãibra, parestesia ou desconforto na musculatura afetada, que aparece num dado nível de esforço deambulatório, podendo aliviar-se com repouso ${ }^{3}$. A CI limita o desempenho físico do indivíduo e leva, conseqüentemente, a comprometimento de atividades funcionais. Distâncias percorridas por indivíduos claudicantes podem estar reduzidas em 50\%, se comparadas às de indivíduos saudáveis de idade similar ${ }^{4,5}$.

Uma das formas de se avaliar a gravidade da DAOP baseia-se no grau de comprometimento funcional do indivíduo claudicante. A literatura descreve protocolos variados de avaliação, tendo como variável de maior interesse o tempo de início de surgimento da dor e de dor máxima limitante durante deambulação ${ }^{1,3-8}$. O teste de caminhada de 6 minutos (T6M) é um teste submáximo de endurance que avalia a distância percorrida num período de 6 minutos. Esse teste tem sido utilizado em pacientes com distúrbios reumatológicos, neurológicos, cardíacos, pulmonares e pediátricos, e sua correlação com a capacidade aeróbica máxima é considerada satisfatória ${ }^{9}$. Este teste, portanto, pode ser usado para fins de comparação da capacidade funcional antes e após intervenções, mas tem a desvantagem de ser um teste cuja velocidade é determinada pelo próprio indivíduo ${ }^{10}$.

Mais recentemente, um novo teste de caminhada no solo vem sendo utilizado para avaliação da capacidade funcional em indivíduos com patologias diversas ${ }^{11}$. Este teste, denominado shuttle walking test, ou teste de deslocamento bidirecional progressivo (TDBP), tem a vantagem de ter a velocidade controlada externamente e impor um esforço progressivo. É um teste de custo baixo e, por ter a intensidade gradualmente aumentada pelo controle externo da velocidade, guarda, portanto, a possibilidade de revelar mais fielmente a capacidade funcional do paciente. Entretanto, o TDBP ainda não é amplamente utilizado em pacientes com CI, e suas características psicométricas nessa população ainda não foram investigadas apropriadamente ${ }^{12}$.

Uma vez que a obstrução arterial periférica pode se apresentar de maneira difusa, com clínica diversa e com resultados de intervenção variados, é fundamental que a avaliação desses pacientes seja feita com instrumentos que possam apresentar dados objetivos e reprodutíveis. A investigação de formas de avaliação simples, mas fidedignas, é importante, sobretudo em áreas cuja restrição econômica inviabiliza recursos tecnológicos mais avançados.

Portanto, o propósito deste estudo piloto foi investigar e contrastar a confiabilidade do T6M com TDBP em indivíduos claudicantes portadores de DAOP.

\section{Métodos}

\section{Sujeitos}

Para condução deste estudo piloto, uma amostragem de conveniência foi selecionada, junto aos participantes do Projeto de Extensão de Atendimento a Portadores de Doenças Vasculares Periféricas do Centro Universitário de Belo Horizonte, para participar do estudo. Pacientes que tivessem DAOP comprovada através do Doppler ou clinicamente e estágio II de Fontai$n \mathrm{e}^{13}$, independente de sexo, etnia ou idade, foram incluídos. Foram excluídos: portadores de DAOP assintomática, dor claudicante de origem não-vascular, angina instável, diabetes não-controlado, hipertensão não-controlada, alterações no eletrocardiograma sugerindo infarto agudo do miocárdio ou outro episódio cardíaco agudo, embolia pulmonar recente e pacientes que apresentaram alguma alteração neuromuscular que limitasse a realização do teste de caminhada.

O estudo foi aprovado pelo Comitê de Ética do Centro Universitário de Belo Horizonte, e não existe conflito de interesse por parte dos autores desta pesquisa. Todos os participantes receberam informações escritas e orais sobre os procedimentos do estudo e se juntaram ao projeto apenas após terem assinado um termo de consentimento livre e esclarecido. 


\section{Procedimentos}

O T6M foi realizado numa pista circular de 60 metros, com demarcações a cada 2 metros ao longo do circuito. $\mathrm{O}$ paciente foi orientado a andar o mais rápido possível sem correr, durante 6 minutos. A cada minuto, foi dado um comando verbal a fim de se estimular o paciente a alcançar a performance mais uniforme possível.

Antes do início do teste, os pacientes foram equipados com um cardiofreqüencímetro (marca Polar ${ }^{\mathrm{TM}}$ ) para registro da freqüência cardíaca (FC) a cada minuto durante o teste. Antes e após a realização do teste, foi aferida a pressão arterial (PA). Os valores utilizados para análise estatística foram aqueles obtidos ao final do teste. A escala de percepção subjetiva de esforço (escala de Borg $)^{14}$ foi utilizada ao final do teste. Caso o paciente necessitasse interromper a caminhada durante o teste por motivo de cansaço ou dor claudicante máxima, eralhe permitido reassumir a caminhada tão logo se recuperasse, a fim de completar os 6 minutos de teste.

Para a realização do TDBP, uma distância de 10 metros foi demarcada no solo com dois cones. O participante foi instruído a dar voltas consecutivas em torno de ambos os cones, com velocidades que aumentaram progressivamente até a exaustão, presença de sintoma claudicante ou incapacidade de manter o ritmo de deslocamento previamente estabelecido. A velocidade de deslocamento é aumentada a cada minuto $(0,17 \mathrm{~m} / \mathrm{s})$ e controlada por sinais de áudio, gerados por aparelho de som portátil. O TDBP consiste de 12 níveis, com duração de 1 minuto cada, sendo que a velocidade inicial é de $0,5 \mathrm{~m} / \mathrm{s}$, até o máximo de $2,37 \mathrm{~m} / \mathrm{s}$.

Os mesmos procedimentos quanto ao registro da $\mathrm{FC}$, da PA e da percepção subjetiva do esforço realizado no T6M foram utilizados no TDBP. Os dois testes de caminhada foram realizados e repetidos em um período máximo de 1 semana. A ordem de aplicação dos testes foi aleatória, e cada teste foi realizado com 20 minutos de intervalo entre si. Antes da coleta efetiva de dados, os pacientes passaram por um período de familiarização com os procedimentos. O tempo de surgimento de dor, registrado em segundos, durante a caminhada, bem como a distância total percorrida, foram registrados em ambos os testes.

\section{Análise estatística}

Os dados foram apresentados como média \pm desvio padrão e intervalo de confiança de $95 \%$. O coeficiente de correlação intraclasse $\left(\mathrm{ICC}_{2,1}\right)$ foi utilizado para avaliação da reprodutibilidade das variáveis de principal interesse neste estudo que foram distância e tempo de caminhada em ambos os testes. O teste $t$ de Student foi utilizado para comparação da diferença entre as médias quando apropriado. Um valor de alfa menor ou igual a 0,05 foi considerado para significância estatística.

\section{Resultados}

Quatorze indivíduos preencheram os critérios de inclusão e foram incluídos neste piloto. Dentre os pacientes contatados, quatorze realizaram ambos os testes, e onze realizaram apenas o TDBP. Na avaliação das médias de distância máxima percorrida, não houve diferença estatisticamente significativa com o $\mathrm{T} 6 \mathrm{M}$ em ambas as avaliações, nem com o TDBP. O ICC $_{2,1}$ demonstrou uma confiabilidade alta e significativa em ambos os testes, sendo que o TDBP apresentou correlação ainda superior ao T6M (Tabela 1). Também não houve diferença estatisticamente significativa entre as distâncias máximas alcançadas entre o T6M e o TDBP, tanto na avaliação 1 quanto na avaliação $2(p=0,36$ e $p$ $=0,35$, respectivamente).

Quanto ao tempo de surgimento de dor inicial, apenas dois pacientes falharam no relato durante o T6M. Os tempos médios, em segundos, não diferiram estatisticamente, embora tenha havido uma tendência à redução no tempo de surgimento de dor inicial na segunda avaliação. A reprodutibilidade dessa medida foi alta $\left(\mathrm{ICC}_{2,1}=0,81\right)$ e significativa (Tabela 2$)$. Já com o TDBP, quatro pacientes falharam em relatar dor inicial. Não houve diferença estatisticamente significativa entre as médias de tempo de surgimento de dor inicial nas duas avaliações realizadas com o TDBP. Tais medidas apresentaram alto índice de confiabilidade teste-reteste $\left(\mathrm{ICC}_{2,1}=0,91\right)$. Os pacientes gastaram um tempo médio em valor absoluto maior para relato de dor inicial durante o TDBP nas avaliações 1 e 2, quando comparado com o T6M ( $\mathrm{p}=0,05 \mathrm{e} \mathrm{p}=0,04$, respectivamente).

Não houve diferença estatisticamente significativa nas médias entre as duas avaliações de FC, pressão arterial sistólica (PAS) e pressão arterial diastólica (PAD), 
Tabela 1 - Distância máxima (em metros) percorrida em ambos os testes (teste-reteste)

\begin{tabular}{ccccccc}
\hline Teste & $\mathbf{n}$ & $\begin{array}{c}\text { Avaliação 1 } \\
(\mathbf{I C 9 5} \%)\end{array}$ & $\begin{array}{c}\text { Avaliação 2 } \\
(\mathbf{I C 9 5} \%)\end{array}$ & $\mathbf{p}^{*}$ & $\mathbf{I C C 2 , 1}$ & $\mathbf{p}^{\dagger}$ \\
\hline \multirow{2}{*}{ T6M } & 14 & $397,04 \pm 120,74$ & $408,60 \pm 153,64$ & 0,58 & $0,87(0,64-0,96)$ & $<0,00005$ \\
& & $(329,08-465,00)$ & $(327,58-488,54)$ & & & \\
TDBP & 11 & $345,00 \pm 140,75$ & $345,91 \pm 125,97$ & 0,92 & $0,99(0,95-0,99)$ & $<0,00005$ \\
& & $(261,82-428,17)$ & $(271,46-420,35)$ & & &
\end{tabular}

$\mathrm{ICC}_{2,1}=$ coeficiente de correlação de intraclasse, modelo 2,$1 ; \mathrm{IC} 95 \%=$ intervalo de confiança de $95 \%$; $\mathrm{n}=$ número de indivíduos testados; T6M = teste de caminhada de 6 minutos; TDBP = teste de deslocamento bidirecional progressivo.

* Nível de significância entre as médias das distâncias entre avaliação 1 e avaliação 2.

${ }^{\dagger}$ Nível de significância do $\mathrm{ICC}_{2,1}$.

Tabela 2 - Tempo de surgimento de sintoma claudicante inicial (em segundos) em ambos os testes (teste-reteste)

\begin{tabular}{ccccccc}
\hline Teste & $\mathbf{n}$ & $\begin{array}{c}\text { Avaliação 1 } \\
(\mathbf{I C 9 5} \%)\end{array}$ & $\begin{array}{c}\text { Avaliação 2 } \\
(\mathbf{I C 9 5} \%)\end{array}$ & $\mathbf{p}^{*}$ & ICC2,1 & $\mathbf{p}^{\dagger}$ \\
\hline \multirow{2}{*}{ T6M } & 12 & $\begin{array}{c}172,25 \pm 88,23 \\
(122,33-222,17)\end{array}$ & $\begin{array}{c}148,58 \pm 70,36 \\
(108,77-188,39)\end{array}$ & 0,13 & $0,81(0,46-0,94)$ & \\
& & & $282,00 \pm 141,90$ & $267,14 \pm 150,58$ & 0,0004 \\
TDBP & 7 & $(176,88-387,12)$ & $(155,59-378,69)$ & & $0,91(0,58-0,98)$ & 0,0008
\end{tabular}

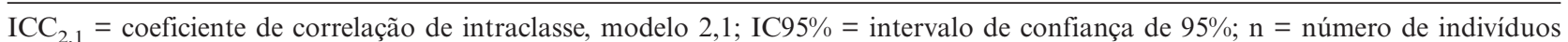
testados; T6M = teste de caminhada de 6 minutos; TDBP = teste de deslocamento bidirecional progressivo.

* Nível de significância entre as médias das distâncias entre avaliação 1 e avaliação 2.

† Nível de significância do $\mathrm{ICC}_{2,1}$ baseado em 12 pares de observação para o T6M e sete pares para o TDBP.

quando comparado o T6M com o TDBP, exceto pela PAD, que apresentou um valor estatisticamente menor na segunda avaliação durante o T6M. Houve, contudo, uma tendência dos valores hemodinâmicos serem maiores com o TDBP em relação à FC (Tabela 3).

\section{Discussão}

O objetivo deste estudo foi contrastar a confiabilidade do T6M com a do TDBP e registrar a performance do indivíduo portador de DAOP através do relato de tempo de início de dor, distância percorrida e estresse cardiovascular. Ambos os testes geraram altos coeficientes de confiabilidade em relação à distância percorrida, embora o TDBP tenha demonstrado maior magnitude de correlação $\left(\mathrm{ICC}_{2,1}=0,99\right)$ em relação ao $\mathrm{T} 6 \mathrm{M}\left(\mathrm{ICC}_{2,1}\right.$ $=0,87$ ). Os resultados obtidos com o T6M corroboram o estudo de Montgomery et al., que descreveram alta confiabilidade e baixo coeficiente de variação (CV) - 0,94 e 10,4\%, respectivamente - ao avaliar a distância percorrida em pacientes com DAOP ${ }^{10}$. Na literatura, os relatos de distância percorrida no T6M variam de $382 \pm 12$ a $433 \pm 11$ metros $^{13,15-17}$, sendo similares aos encontrados em nosso estudo na população estudada.

O TDBP não tem sido universalmente utilizado para avaliação da performance de portadores de DAOP. Devido a esse fato, há uma restrição de dados na literatura sobre a performance dessa população no TDBP, limitando, em parte, a discussão dos resultados obtidos nesse estudo com esse teste. Num estudo envolvendo o TDBP, Zwierska et al. ${ }^{18}$ registraram uma média de distância de caminhada até sintoma limitante de $217 \pm 17$ metros após três avaliações, com altos índices de confiabilidade $(0,88)$ e CV próximo a $16 \%$. Tais valores são, contudo, menores em relação aos demonstrados no presente estudo. 
Tabela 3 - Comportamento das variáveis hemodinâmicas nos testes de caminhada

\begin{tabular}{|c|c|c|c|c|c|c|}
\hline & \multicolumn{3}{|c|}{ T6M $(n=14)$} & \multicolumn{3}{|c|}{$\operatorname{TDBP}(n=11)$} \\
\hline & $\begin{array}{l}\text { Avaliação } 1 \\
\text { (IC95\%) }\end{array}$ & $\begin{array}{c}\text { Avaliação } 2 \\
\text { (IC95\%) }\end{array}$ & $\mathbf{p}^{*}$ & $\begin{array}{l}\text { Avaliação } 1 \\
\text { (IC95\%) }\end{array}$ & $\begin{array}{c}\text { Avaliação } 2 \\
\text { (IC95\%) }\end{array}$ & $\mathbf{p}^{*}$ \\
\hline FC & $\begin{array}{c}103,57 \pm 11,68 \\
(97,45-109,69)\end{array}$ & $\begin{array}{c}109,93 \pm 17,06 \\
(101,00-118,86)\end{array}$ & 0,07 & $\begin{array}{c}114,27 \pm 17,14 \\
(104,15-124,40)\end{array}$ & $\begin{array}{c}113,00 \pm 16,79 \\
(103,08-122,02)\end{array}$ & 0,22 \\
\hline PAS & $\begin{array}{c}157,14 \pm 25,25 \\
(143,92-170,37)\end{array}$ & $\begin{array}{c}153,21 \pm 23,50 \\
(140,90-165,52)\end{array}$ & 0,25 & $\begin{array}{c}146,36 \pm 20,26 \\
(134,39-158,34)\end{array}$ & $\begin{array}{c}158,10 \pm 81,36 \\
(145,56-170,81)\end{array}$ & 0,09 \\
\hline PAD & $\begin{array}{c}83,21 \pm 12,07 \\
(76,91-89,51)\end{array}$ & $\begin{array}{l}79,14 \pm 11,01 \\
(73,32-84,96)\end{array}$ & 0,04 & $\begin{array}{l}80,45 \pm 10,11 \\
(74,48-86,43)\end{array}$ & $\begin{array}{c}79,09 \pm 8,31 \\
(74,18-84,00)\end{array}$ & 0,57 \\
\hline
\end{tabular}

$\overline{\mathrm{FC}}=$ freqüência cardíaca (batimentos por minuto); IC95\% = intervalo de confiança de 95\%; $\mathrm{n}=$ número de indivíduos testados; PAS = pressão arterial sistólica $(\mathrm{mmHg}) ; \mathrm{PAD}=$ pressão arterial diastólica $(\mathrm{mmHg}) ; \mathrm{T} 6 \mathrm{M}=$ teste de caminhada de 6 minutos; TDBP = teste de deslocamento bidirecional progressivo.

* Nível de significância entre as médias das variáveis hemodinâmicas do T6M e TDBP.

O tempo de dor inicial, observado em diferentes protocolos, vem sendo relatado na literatura com grande variabilidade em relação a outras variáveis. Em um estudo com pacientes claudicantes utilizando o T6M, $69 \%$ dos 64 pacientes estudados relataram dor inicial, sendo registrado um tempo médio de $135 \pm 59$ segundos e moderada correlação teste-reteste $\left(\mathrm{ICC}_{2,1}=0,75 \mathrm{e} \mathrm{CV}\right.$ $=47 \%)^{9}$. Outros estudos relataram alto coeficiente de correlação $(0,95)$ em relação ao tempo de dor inicial no $\mathrm{T}_{6} \mathrm{M}^{5}$. Walker et al. relatam alta confiabilidade testereteste no tempo e distância de início de dor com $\mathrm{TDBP}^{12}$. No presente estudo, o coeficiente de correlação no tempo de início de dor no T6M foi elevado $\left(\mathrm{ICC}_{2,1}=0,81\right)$, porém o coeficiente de correlação do TDBP mostrou-se superior $\left(\mathrm{ICC}_{2,1}=0,91\right)$.

Os testes de caminhada são utilizados mais freqüentemente para avaliar a performance do paciente antes e após intervenção. Como a base da reabilitação em pacientes com DAOP é a deambulação, os resultados obtidos através dos testes (distância, velocidade, tempo de surgimento de sintoma) podem ser úteis para prescrever a intensidade específica para a atividade e potencializar a reabilitação desses indivíduos. Com o T6M, não é possível estipular precisamente a velocidade de caminhada, devido ao fato de o paciente poder exercer maior controle sobre ela durante sua performance. Já com o TDBP, como a velocidade é determinada externamente e o paciente exerce menos controle sobre sua performance, é possível estipular a velocidade exata do aparecimento dos sintomas, e, portanto, os resultados obtidos com esse teste tornam-se parâmetros mais confiáveis para a prescrição da intensidade da reabilitação.

Tem-se sugerido que o TDBP imponha um maior estresse cardiovascular por ser progressivo e sintomalimitado, em comparação a outros testes de caminhada com características submáximas, como o $\mathrm{T} 6 \mathrm{M}^{19}$. Os dados encontrados neste estudo não confirmaram essa sugestão, uma vez que não houve diferença estatisticamente significativa nas variáveis hemodinâmicas entre os dois testes, com exceção da PAD durante o T6M. Essa diferença não se apresenta, contudo, importante do ponto de vista clínico. Houve uma tendência da FC em apresentar-se maior durante o TDBP.

Vaggagini et al. compararam o T6M com o TDBP nos pacientes com DPOC e não verificaram que o TDBP tenha imposto sobrecarga cardiorrespiratória mais elevada aos pacientes ${ }^{20}$. Em outro estudo, em pacientes com DPOC, as distâncias obtidas no T6M e no TDBP foram similares; no entanto, no TDBP, as correlações geradas com os parâmetros cardiorrespiratórios foram fortes e significativas, levando os autores a concluir que o TDBP 
é mais confiável que o T6M para avaliar o desempenho dos pacientes com DPOC ${ }^{21}$.

\section{Conclusão}

Este estudo indica que ambos os testes de caminhada são confiáveis e úteis para avaliação clínicofuncional de pacientes de CI secundária a DAOP. O TDBP, entretanto, gerou índices de confiabilidade mais elevados no teste-reteste, podendo ser uma melhor opção para avaliação da performance desses indivíduos. Deve-se ressaltar que esta pesquisa prima pela investigação da confiabilidade de medidas e, portanto, não tem como objetivo verificar diferenças entre os testes. A generalização dos resultados encontrados é limitada pelo tamanho da amostra; desse modo, para aumentar a universalidade dos resultados, uma nova pesquisa deveria ser conduzida com maior tamanho de amostra.

\section{Referências}

1. Prieto AC. Doença arterial obstrutiva periférica (DAOP). Disponível em: http://www.transdoreso.org/ estudo_cardiologia.shtml.

2. Dormandy JA, Rutherford RB. Management of peripheral arterial disease (PAD). TransAtlantic inter-society consensus (TASC) working group. J Vasc Surg. 2000;31:(Suppl 1):S513.

3. Borges JL. Doença arterial obstrutiva periférica no idoso. In: Borges JL, editor. Manual de cardiogeriatria. $2^{\mathrm{a}}$ Ed. São Paulo: BBS; 2005. p. 83-94.

4. Stewart KJ, Hiatt WR, Regensteiner JG, Hirsch AT. Exercise training for claudication. N Engl J Med. 2002;347:194151 .

5. Gardner AW, Skinner JS, Vaughan NR, Bryant CX, Smith LK. Comparison of three progressive exercise protocols in peripheral vascular occlusive disease. Angiology. 1992;43:66171.

6. Green S. Haemodynamic limitations and exercise performance in peripheral arterial disease. Clin Physiol Funct Imaging. 2002;22:81-91.

7. Labs KH, Nehler MR, Roessner M, Jaeger KA, Hiatt WR. Reliability of treadmill testing in peripheral arterial disease: a comparison of a constant load with a graded load treadmill protocol. Vasc Med. 1999;4:239-46.

8. Cachovan M, Rogatti W, Woltering F, et al. Randomized reliability study evaluating constant-load and graded-exercise treadmill test for intermittent claudication. Angiology. 1999;50:193-200.

9. Solway S, Brooks D, Lacasse Y, Thomas S. A qualitative systematic overview of the measurement properties of functional walk tests used in the cardiorespiratory domain. Chest. 2001;119:256-70.
10. Montgomery PS, Gardner AW. The clinical utility of a sixminute walk test in peripheral arterial occlusive disease patients. J Am Geriatr Soc. 1998;46:706-11.

11. Singh SJ, Morgan MD, Scott S, Walters D, Hardman AE. Development of a shuttle walking test of disability in patients with chronic airways obstruction. Thorax. 1992;47:1019-24.

12. Walker RD, Nawaz S, Wilkinson CH, Saxton JM, Pockley AG, Wood RF. Influence of upper- and lower-limb exercise training on cardiovascular function and walking distances in patients with intermittent claudication. J Vasc Surg. 2000;31:662-9.

13. Gardner AW, Katzel LI, Sorkin JD, et al. Exercise rehabilitation improves functional outcomes and peripheral circulation in patients with intermittent claudication: a randomized controlled trial. J Am Geriatr Soc. 2001;49:755-62.

14. Dawes HN, Barker KL, Cockburn J, Roach N, Scott O, Wade D. Borg's rating of perceived exertion scales: do the verbal anchors mean the same for different clinical groups? Arch Phys Med Rehabil. 2005;86:912-6.

15. Gardner AW, Montgomery PS, Flinn WR, Katzel LI. The effect of exercise intensity on the response to exercise rehabilitation in patients with intermittent claudication. J Vasc Surg. 2005;42:702-9.

16. Atkins LM, Gardner AW. The relationship between lower extremity functional strength and severity of peripheral arterial disease. Angiology. 2004;55:347-55.

17. McDermott MM, Liu K, Greenland P, et al. Functional decline in peripheral arterial disease: associations with the ankle brachial index and leg symptons. JAMA. 2004;292:45361.

18. Zwierska I, Nawaz S, Walker RD, Wood RF, Pockley AG, Saxton JM. Treadmill versus Shuttle Walk Tests of Walking Ability in Intermittent Claudication. Med Sci Sports Exerc. 2004;36:1835-40.

19. Lewis ME, Newall C, Townend JN, Hill SL, Bonser RS. Incremental shuttle walk test in the assessment of patients for heart transplantation. Heart. 2001;86:183-7.

20. Vagaggini B, Taccola M, Severino S, et al. Shuttle walking test and 6-minute walking test induce a similar cardiorespiratory performance in patients recovering from an acute exacerbation of chronic obstructive pulmonary disease. Respiration. 2003;70:579-84.

21. Onorati P, AntonuICC R, Valli G, et al. Non-invasive evaluation of gas exchange during a shuttle walking test vs. a 6-min walking test to assess exercise tolerance in COPD patients. Eur J Appl Physiol. 2003;89:331-6.

Correspondência:

Danielle Aparecida Gomes Pereira

Rua João Gualberto Filho, 1260/604, Bairro Sagrada Família

CEP 31035-570 - Belo Horizonte, MG

Tel.: (31) 3309.9137, (31) 9103.7415

E-mail: d.fisio@ig.com.br 